\title{
Extension of the Mechanistic Tissue Distribution Model of Rodgers and Rowland by Systematic Incorporation of Lysosomal Trapping: Impact on Unbound Partition Coefficient and Volume of Distribution Predictions in the Rat ${ }^{\text {\} }$
}

\author{
Maximilian V. Schmitt, Andreas Reichel, Xiaohui Liu, Gert Fricker, and Philip Lienau \\ Bayer AG, Pharmaceuticals R\&D, Translational Sciences, Research Pharmacokinetics, Berlin, Germany (M.V.S., A.R., P.L.); School \\ of Life Sciences, Tsinghua University, Beijing, China (X.L.); and Institute of Pharmacy and Molecular Biotechnology, University of \\ Heidelberg, Heidelberg, Germany (M.V.S., G.F.)
}

Received June 26, 2020; accepted October 28, 2020

\begin{abstract}
Physiologically based pharmacokinetic modeling has become a standard tool to predict drug distribution in early stages of drug discovery; however, this does not currently encompass lysosomal trapping. For basic lipophilic compounds, lysosomal sequestration is known to potentially influence intracellular as well as tissue distribution. The aim of our research was to reliably predict the lysosomal drug content and ultimately integrate this mechanism into pharmacokinetic prediction models. First, we further validated our previously presented method to predict the lysosomal drug content (Schmitt et al., 2019) for a larger set of compounds $(n=41)$ showing a very good predictivity. Using the lysosomal marker lipid bis(monoacylglycero)phosphate, we estimated the lysosomal volume fraction for all major tissues in the rat, ranging from $0.03 \%$ for adipose up to $5.3 \%$ for spleen. The $\mathrm{pH}$-driven lysosomal trapping was then estimated and fully integrated into the mechanistic distribution model published by Rodgers et al. (2005). Predictions of $\mathrm{Kpu}$ improved for all lysosome-rich tissues. For instance, Kpu
\end{abstract}

increased for nicotine 4-fold (spleen) and 2-fold (lung and kidney) and for quinidine 1.8-fold (brain), although for most other drugs the effects were much less ( $\leq 7 \%)$. Overall, the effect was strongest for basic compounds with a lower lipophilicity, such as nicotine, for which the unbound volume of distribution at steady-state prediction changed from 1.34 to $1.58 \mathrm{l} / \mathrm{kg}$. For more lipophilic (basic) compounds or those that already show strong interactions with acidic phospholipids, the additional contribution of lysosomal trapping was less pronounced. Nevertheless, lysosomal trapping will also affect intracellular distribution of such compounds.

\section{SIGNIFICANCE STATEMENT}

The estimation of the lysosomal content in all body tissues facilitated the incorporation of lysosomal sequestration into a general physiologically based pharmacokinetic model, leading to improved predictions as well as elucidating its influence on tissue and subcellular distribution in the rat.

\section{Introduction}

Many basic lipophilic drugs exhibit a deep distribution that is reflected in high volumes of distribution (Vss). The partitioning into individual tissues can however differ considerably, and it is not possible to deduce this from the volume of distribution. Furthermore, experimental determination of tissue partition coefficients $(\mathrm{Kp})$ is not a standard in drug discovery, as it is very resource-intensive. Therefore, the development of mechanistic models that are based on the tissue composition of the body and the physicochemical properties of the drug was instrumental to enable early estimation of drug distribution in drug discovery and development.

This work was financed by Bayer AG, Germany.

Primary laboratory of origin: Dr. Maximilian V. Schmitt, Bayer AG, Berlin, Germany

https://doi.org/10.1124/dmd.120.000161.

S This article has supplemental material available at dmd.aspetjournals.org.
Poulin and Theil (2000) pioneered the mathematical description of partition processes into tissue water, proteins, and neutral lipids, focusing on the neutral drug molecules. Later, Rodgers and Rowland expanded the tissue composition-based distribution equations by inclusion of drug ionization and the resulting interaction of the charged drug species with proteins and membrane constituents (Rodgers et al., 2005; Rodgers and Rowland, 2006). In these models, the dominant affinity of the charged species of basic drugs $\left(\mathrm{p} K_{\mathrm{a}}>7\right)$ is considered to be acidic phospholipids in the tissue (Yata et al., 1990). Predictions for neutral and acidic drugs are far more accurate than for basic drugs (Chan et al., 2018), suggesting further processes being involved in the distribution of bases that so far are not accounted for. A potential additional mechanism is extensive $\mathrm{pH}$-driven sequestration into lysosomes, which can lead to enormous concentrations within the lysosome [up to 160,000-fold compared with the cytosol (MacIntyre and Cutler, 1988a,b)]. In our previous work, we have shown the huge impact this has on the intracellular distribution in rat hepatocytes, with lysosomes

ABBREVIATIONS: AP, acidic phospholipid; BMP, bis(monoacylglycero)phosphate; B:P ratio, blood-to-plasma ratio; EW, extracellular water; IW, intracellular water; Kp, partition coefficient; Kpu, unbound partition coefficient; LC-MS/MS Liquid Chromatography-Mass Spectroscopy-Mass SpectroscopyNL, neutral lipid; NP, neutral phospholipid; PK Pharmacokinetic PKI, protein kinase inhibitor; Vss, volume of distribution at steady state; Vuss, unbound volume of distribution at steady state. 
holding $>50 \%$ of the intracellular drug (Schmitt et al., 2019): lysosomal trapping may therefore also have the potential to influence overall tissue distribution and may further improve predictions of tissue distribution for basic drugs.

Although lysosomal abundance has been qualitatively known in some tissues for quite some time (de Duve et al., 1974; Blouin et al., 1977), the parameterization needed for a physiologically based pharmacokinetic model is still challenging, as their size is highly variable in different cell types (Bandyopadhyay et al., 2014). There has been a first approach to incorporate lysosomal sequestration in lung, kidney, and liver using published data on lysosomal volume fractions and $\mathrm{pH}$ of their main cell types (Assmus et al., 2017). This model can only be applied to well studied tissues and does not cover other lysosome-rich tissues, such as spleen, brain, and gut. To be able to include lysosomal trapping in all body tissues, a surrogate for the lysosomal size is required, e.g. by a marker that is unique for lysosomes. BMP, a lipid exclusively found in the luminal side of the endo-/lysosomal membrane, is responsible for endo-/lysosomal stabilization and fusion, and it promotes hydrolysis by enhancing adhesion of enzymes and activator proteins to the inner lysosomal membrane and cholesterol transport (Schulze et al., 2009; Gallala and Sandhoff, 2011; Hullin-Matsuda et al., 2014). The involvement in many essential lysosomal processes makes its abundance a good surrogate for the size of the endo-/lysosomal system. We have recently published BMP concentrations in all major rat tissues (Wang and Schmitt et al., 2019), thereby providing reliable estimates of the size of the lysosomal system in these tissues. This knowledge allows for a new possibility to include lysosomal trapping into overall tissuedistribution predictions.

The aim of this study was to further expand the mechanistic equations of Rodgers et al. (2005) by inclusion of lysosomal sequestration as an additional distribution process for basic drugs $\left(\mathrm{p} K_{\mathrm{a}}>7\right)$. We have validated our previously published approach to predict the intracellular drug distribution to lysosomes in rat hepatocytes (Schmitt et al., 2019) for a broad set of compounds, estimated the size of the lysosomal compartment in various rat tissues, and integrated both into the mechanistic equations to predict tissue partitioning. Impact on $\mathrm{Kpu}$ was evaluated, comparing prediction results to the original model and experimental data of the original data set of 28 basic compounds (Rodgers et al., 2005). The in vivo distribution of 13 protein kinase inhibitors (PKIs), extending the physicochemical property space of the original data set, was used to further investigate the performance of both models and to evaluate the influence of lysosomal trapping on $\mathrm{Kpu}$ and Vuss.

\section{Materials and Methods}

\section{Chemicals and Reagents}

Afatinib, axitinib, bosutinib, cediranib, crizotinib, dasatinib, erlotinib, gefitinib, ibrutinib, lapatinib, linsitinib, masitinib, motesanib, nilotinib, nintedanib, olaparib, pazopanib, quizartinib, saracatinib, selumetinib, sunitinib, tandutinib, and vandetanib were purchased from Selleck Chemicals LLC (Houston, TX). Imatinib was purchased from Enzo Life Sciences AG (Lausen, Germany), and regorafenib was obtained from Bayer AG (Berlin, Germany). Monensin sodium was bought from Sigma-Aldrich (St. Louis, MO). Acetonitrile and methanol were purchased from Honeywell Specialty Chemicals Seelze GmbH (Seelze, Germany).

\section{Animals and Treatment}

Male Han:Wistar rats (Envigo, Netherlands/Janvier Laboratories, France) of $300 \pm 30 \mathrm{~g}$ body weight were used. Animals were housed in groups of up to three in transparent standard cages at $22^{\circ} \mathrm{C}$ with a 12 -hour light/dark cycle. Standard rat diet and water were provided ad libitum.

\section{Direct Quantification of Lysosomal Drug Content}

The lysosomal drug content was measured in rat hepatocytes as described in Schmitt et al. (2019). In short, rat hepatocytes were isolated, purified, seeded on 24-well collagen-coated plates and subsequently incubated for 24 hours, resulting in greatly reduced transporter expression as well as metabolic enzyme activity. Drug accumulation of compounds was measured 1) in cultured control hepatocytes and 2) in hepatocytes with inactivated lysosomes ( $+25 \mu \mathrm{M}$ monensin). Samples were analyzed via LC-MS/MS, and the lysosomal drug content was calculated by the difference of accumulation in the cells. The lysosomal drug content was measured for a set of 26 test compounds at a low concentration of $5 \mu \mathrm{M}$ to avoid lysosomal saturation during the experiments (Schmitt et al., 2019). Experimental determination of the lysosomal drug content was done extensively $(n=9)$ for nine compounds with expected low, medium, and high potential for lysosomal trapping; other compounds were each measured twice.

\section{Prediction of Lysosomal Drug Sequestration}

The extent of lysosomal sequestration $L$ for drugs was calculated as previously described in Schmitt et al. (2019) using the following equation:

$$
L=\int_{p H_{\min }}^{p H_{c y t o}} \frac{V(p H) \cdot K_{L}\left(p H, p K_{a, 1}, p K_{a, 2}\right)}{V_{B}} d p H,
$$

with $V(p H)$ as the volume of the lysosomal compartment at a given $\mathrm{pH}, K_{L}$ as the concentration ratio of compound between the cytosol and the lysosome depending on the lysosomal $\mathrm{pH}$ from $\mathrm{pH}_{\min }=4$ up to $\mathrm{pH}_{\text {cyto }}=7.2$, as well as the most and second most basic drug moiety as described by MacIntyre and Cutler (1988a). $V_{B}$ represents the volume of the nonacidic compartment in a hepatocyte and $\mathrm{dpH}$ defines that the $\mathrm{pH}$ range needs to be integrated. The extent of lysosomal sequestration $L$ was expressed as the percentage in lysosomes of the total drug in the cells. Correlation of predictions and experimental results were carried out in OriginPro 2018.

\section{Size of the Endo-/Lysosomal System in Rat Tissues}

The fractional volume of lysosomes in the liver was estimated from the extensively characterized rat hepatocytes (Schmitt et al., 2019) under the simplification of hepatocytes being the only cell type in this tissue, as they make up about $93 \%$ of the cellular tissue in the liver (Blouin et al., 1977). Considering the cellular space makes up $84.1 \%$ of the liver (Blouin et al., 1977), a fractional tissue volume of $1.3 \%$ could be calculated for lysosomes. Lysosomal abundance for other tissues was calculated relative to the liver based on the concentration of the lysosome-specific marker lipid BMP (Schulze et al., 2009; Gallala and Sandhoff, 2011; Hullin-Matsuda et al., 2014), which was recently determined by Wang and Schmitt et al. (2019) (Table 2). As BMP levels of bone, pancreas, and thymus were not measured, no lysosomal compartment could be calculated for these tissues.

\section{Lysosomal Sequestration in the Prediction of Drug Tissue Distribution}

The proposed equation to predict tissue distribution of basic lipophilic drugs of Rodgers et al. (2005) considers the $\mathrm{pH}$-driven accumulation in the intracellular water (IW), distribution to the extracellular water (EW), and the association to neutral lipids (NLs)/neutral phospholipids (NPs) and acidic phospholipids $\left(\mathrm{AP}^{-}\right)$:

$$
\begin{aligned}
K p u_{R \& R}=( & \left.\frac{1+10^{p K_{a}-p H_{I W}}}{1+10^{p K_{a}-p H_{p}}} \cdot f_{I W}\right)+f_{E W}+\left(\frac{K_{a} \cdot\left[A P^{-}\right]_{T} \cdot 10^{p K_{a}-p H_{I W}}}{1+10^{p K_{a}-p H_{p}}}\right) \\
& +\left(\frac{P \cdot f_{N L}+(0.3 P+0.7) \cdot f_{N P}}{1+10^{p K_{a}-p H_{p}}}\right)
\end{aligned}
$$

with $f_{I W}, f_{E W}, f_{N L}, f_{N P}$ as fractional volumes of the tissue, $p H_{I W}=7.0$ and $p H_{p}=$ 7.4 as the $\mathrm{pH}$ of the intracellular water and the plasma, $p K_{a}$ as the compound's acid dissociation constant, and $K_{a}$ as the association constant of the compound to acidic phospholipids with the tissue concentration $\left[A P^{-}\right]_{T} . K_{a}$ is estimated from the compound's blood-to-plasma ratio, as described by Rodgers et al. (2005).

With the absence of active transport processes and the steady-state condition in the model, the concentration of the unprotonated base is equal in each compartment, thus allowing the lysosomes to be put into a direct relation to the plasma for estimating the $\mathrm{pH}$-driven sequestration as follows: 


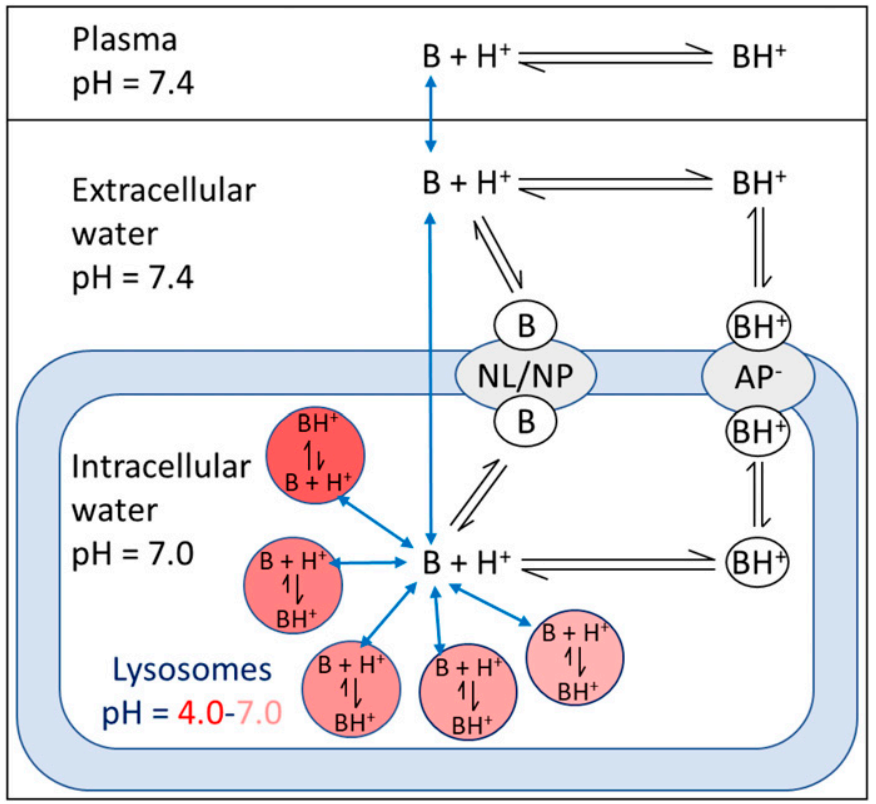

Fig. 1. Schematic illustration of processes included in the mechanistic eq. 3 to predict tissue distribution of basic lipophilic drugs. The tissue distribution is estimated considering the sequestration into lysosomes, which is estimated based on the full $\mathrm{pH}$ profile in hepatocytes, acidic phospholipid binding, neutral (phospho) lipid binding, and distribution into aqueous spaces. B, Base; NP, neutral phospholipid.

$$
\begin{aligned}
\mathrm{Kpu}=\left(\frac{1+10^{p K_{a}-p H_{I W}}}{1+10^{p K_{a}-p H_{p}}} \cdot f_{I W}\right)+f_{E W}+\left(\frac{K_{a} \cdot\left[A P^{-}\right]_{T} \cdot 10^{p K_{a}-p H_{I W}}}{1+10^{p K_{a}-p H_{p}}}\right) \\
+\left(\frac{P \cdot f_{N L}+(0.3 P+0.7) \cdot f_{N P}}{1+10^{p K_{a}-p H_{p}}}\right)+\left(\frac{1+10^{p H_{a}-p H_{L}}}{1+10^{p K_{a}-p H_{p}}} \cdot f_{L y s o}\right)
\end{aligned}
$$

with $f_{L y s p}$ as the fractional volume of lysosomes in the respective tissues and $p H_{L}=5.3$ as an effective $\mathrm{pH}$ across all lysosomes, derived from eq. 1 for monobasic drugs under consideration of the complete $\mathrm{pH}$ profile in rat hepatocytes. All processes accommodated in the mechanistic equation are depicted in Fig. 1.

The unbound volume of distribution was calculated according to Rodgers and Rowland (2007) by:

$$
V u_{s s}=\frac{V_{P}}{f u}+\sum V_{T, i} \cdot K p u_{i},
$$

with $V p$ as the plasma volume, $V u_{s s}$ as the unbound volume of distribution at steady state, $f u$ as the unbound fraction of drug in plasma, $V_{T, i}$ as the volume of individual tissues, and $K p u_{i}$ as the partitioning in tissues, defined as the ratio of the total concentration in the tissue to the unbound plasma concentration, calculated by eq. 3 .

\section{Tissue-Specific Input Parameters}

Parameters to predict the unbound tissue partitioning and the unbound volume of distribution in rats were adopted from Rodgers et al. (2005) and Rodgers and Rowland (2007), with the exception of the intracellular water, which was reduced by the newly defined lysosomal compartment (Table 1).

\section{Compound-Specific Parameters}

Parameters for the original set of compounds by Rodgers and Rowland were taken from the literature (Rodgers and Rowland, 2007). Timolol was excluded from the comparison, as our model is only valid for monobasic compounds, and Rodgers and Rowland treated it as a dibasic compound, i.e., with two highly basic $\mathrm{p} K_{\mathrm{a}}$ values.

The acid dissociation constants of basic drug moieties and the drug lipophilicity of protein kinase inhibitors were predicted in silico using ADMET Predictor (Simulations Plus Inc.). Blood-to-plasma ratio (B:P ratio), unbound fraction in plasma, and in vivo Vss were determined experimentally unless published elsewhere.

B:P Ratio. Blood of Han:Wistar rats was drawn with K-EDTA as an anticoagulant prior to the experiment. The fresh blood of three individual rats was pooled and kept at $37^{\circ} \mathrm{C}$. Blood working solutions were prepared from DMSO stock solutions with final concentrations of 0.3 and $3 \mu \mathrm{M}$, which were subsequently incubated for 15 minutes at $37^{\circ} \mathrm{C}$. Samples of blood $(n=3)$ were drawn for LC-MS/MS analysis. Plasma was obtained by centrifuging the blood at

TABLE 1

\begin{tabular}{|c|c|c|c|c|c|c|c|}
\hline \multirow{2}{*}{ Tissue } & \multicolumn{5}{|c|}{ Fractional Tissue Volume $^{a}$} & \multirow{2}{*}{$\begin{array}{l}\text { Tissue Concentration } \\
\text { of AP }\end{array}$} & \multirow{2}{*}{$\begin{array}{c}\text { Tissue } \\
\text { Volume }\end{array}$} \\
\hline & $\mathrm{NL}$ & NP & EW & $\mathrm{IW}^{b}$ & Lysosomes $^{c}$ & & \\
\hline Ref. & {$\left[{ }^{d}\right]$} & {$\left[{ }^{d}\right]$} & {$\left[{ }^{d}\right]$} & & & $\begin{array}{c}m g / g \\
{\left[{ }^{d}\right]}\end{array}$ & $\begin{array}{l}m l \\
{\left[{ }^{e}\right]}\end{array}$ \\
\hline Blood cells & 0.0017 & 0.0029 & n.a. & 0.603 & n.a. & 0.5 & \\
\hline Adipose & 0.853 & 0.0016 & 0.135 & 0.017 & 0.00027 & 0.4 & 10.43 \\
\hline Bone & 0.017 & 0.0017 & 0.1 & 0.346 & n.d. & 0.67 & 16.47 \\
\hline Brain & 0.039 & 0.0015 & 0.162 & 0.606 & 0.0136 & 0.4 & 1.3 \\
\hline Gut & 0.038 & 0.0125 & 0.282 & 0.463 & 0.0122 & 2.41 & 11.1 \\
\hline Heart & 0.014 & 0.0111 & 0.32 & 0.453 & 0.0032 & 2.25 & 1.08 \\
\hline Kidney & 0.012 & 0.0242 & 0.273 & 0.466 & 0.0173 & 5.03 & 2.85 \\
\hline Liver & 0.014 & 0.024 & 0.161 & 0.560 & 0.0125 & 4.56 & 14.67 \\
\hline Lung & 0.022 & 0.0128 & 0.336 & 0.431 & 0.0151 & 3.91 & 1.53 \\
\hline Muscle & 0.01 & 0.0072 & 0.118 & 0.629 & 0.0011 & 1.53 & 136.24 \\
\hline Pancreas & 0.041 & 0.0093 & 0.12 & 0.664 & n.d. & 1.67 & 0.94 \\
\hline Skin & 0.06 & 0.0044 & 0.382 & 0.289 & 0.0016 & 1.32 & 43.69 \\
\hline Spleen & 0.0077 & 0.0113 & 0.207 & 0.526 & 0.0527 & 3.18 & 0.83 \\
\hline Thymus & 0.017 & 0.0092 & 0.15 & 0.626 & n.d. & 2.3 & 0.73 \\
\hline Plasma & n.a. & n.a. & n.a. & n.a. & n.a. & n.a. & 8.13 \\
\hline
\end{tabular}

Tissue-specific input parameters to predict the unbound tissue partitioning and unbound volume of distribution (Vuss) in rats, which comprises the fractional tissues volumes of Rodgers et al. (2005) extended by a lysosomal compartment

n.a., not available; n.d., not determined.

${ }^{a}$ Based on the tissue wet weight.

${ }^{b}$ Fractional tissue volume of intracellular water of Rodgers et al. (2005) reduced by new lysosomal compartment.

${ }^{c}$ Calculated by subtracting the fractional volume of extracellular water of the liver from total tissue volume and subsequently multiplying by the previously determined fractional volume of lysosomes in hepatocytes (Schmitt et al., 2019). Fractional volumes of tissues were scaled from liver by their BMP content.

${ }^{d}$ Rodgers et al. (2005)

${ }^{e}$ Rodgers and Rowland (2007) 
A

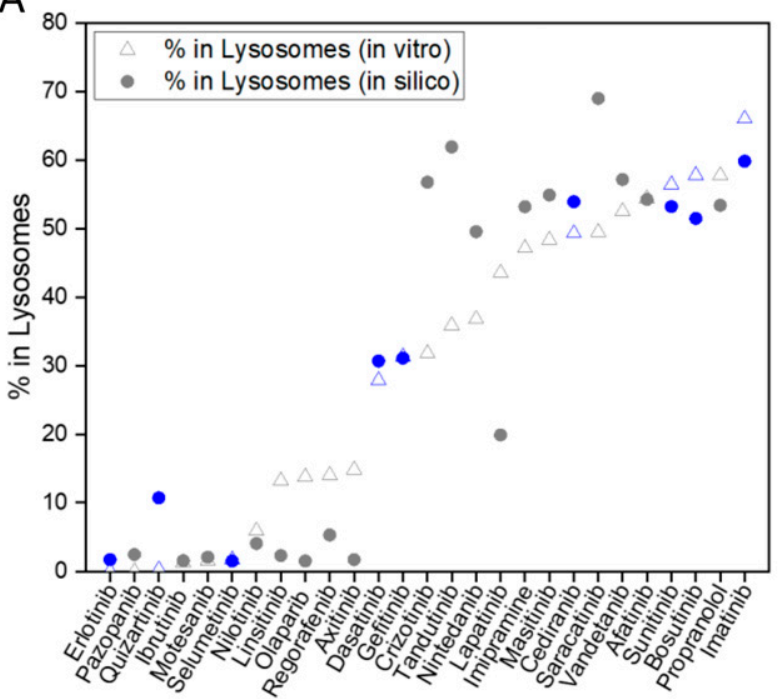

B

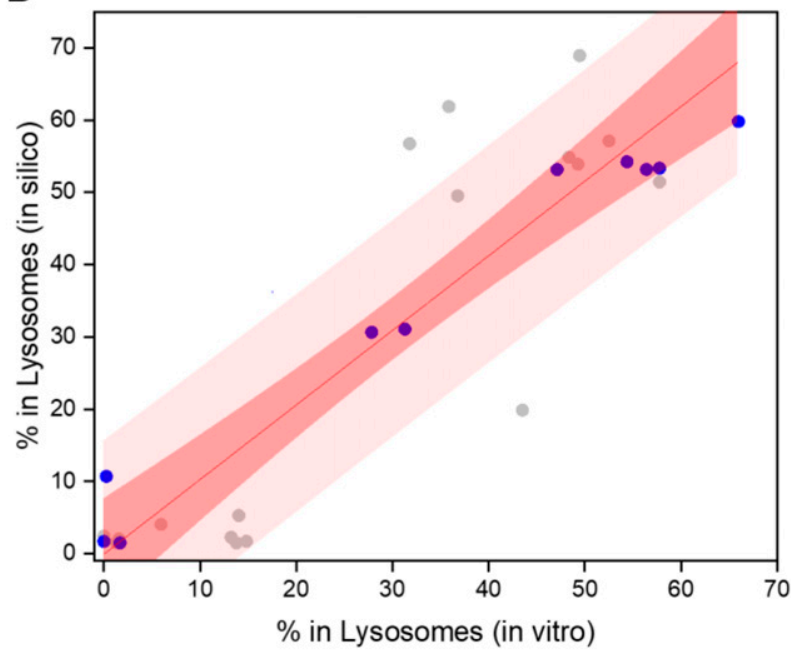

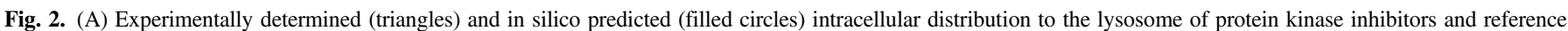

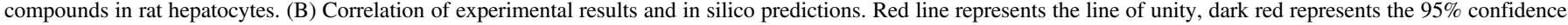

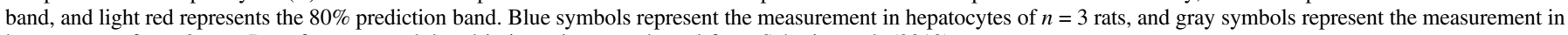
hepatocytes of $n=2$ rats. Data for propranolol and imipramine are adopted from Schmitt et al. (2019).

$3700 \mathrm{rpm}$ for 15 minutes, and samples $(n=3)$ were drawn for LC-MS/MS analysis. Four times the volume of methanol/internal standard was added to each sample for protein precipitation prior to analysis. The mean B:P ratio was calculated from plasma and blood samples from both working concentrations.

Plasma Protein Binding. Plasma protein binding was measured by equilibrium dialysis according to Banker et al. (2003), with slight changes. In short, drugs were diluted into pooled rat plasma $(n=4)$ and dialysis buffer $(n=3)$ to final concentrations of $3 \mu \mathrm{M}$ for the equilibrium dialysis and into plasma vials $(n=3)$ to investigate compound stability. Samples were drawn after 7 hours of incubation at $37^{\circ} \mathrm{C}$. Four times the volume of methanol was added for protein precipitation. Samples were centrifuged at $3700 \mathrm{rpm}$ for 15 minutes and subsequently analyzed via LC-MS/MS. Volume shift was corrected for by a factor of 1.09 for all compounds.

\section{In Vivo Pharmacokinetic Studies}

To reduce animal experiments to a minimum, rat volume of distribution was gathered primarily from assessment reports of the Food and Drug Administration, European Medicines Agency, and Therapeutic Goods Administration, as well as from scientific articles.

The in vivo PK studies of cediranib, imatinib, masitinib, and tandutinib were carried out in catheterized Han:Wistar rats $(n=3)$. Application solutions were prepared in either plasma or diluted PEG400-based formulations. The doses of $0.3-0.5 \mathrm{mg} / \mathrm{kg}$ were administered intravenously into the tail vein. Heparinized blood samples were drawn after 2, 8, 15, 30, and 45 minutes and 1, 2, 4, 7, and 24 hours, and plasma was generated by centrifugation at $3700 \mathrm{rpm}$ for 5 minutes. Samples $\leq 60$ minutes and the application solution were additionally diluted 1:10 in rat plasma. A calibration curve was prepared in the range of $0.25 \mathrm{nM}$ to $5 \mu \mathrm{M}$. To each sample, five times the volume of acetonitrile containing internal standard was added and subsequently centrifuged at $3700 \mathrm{rpm}$ for 15 minutes prior to LCMS/MS analysis. Pharmacokinetic calculations were done with WinNonlin (version 6.1; Phoenix).

\section{Analytical Method}

Samples were analyzed with liquid chromatography-tandem mass spectrometry using an Agilent 1290 Infinity System comprising a G4220A binary pump, a G1316C column compartment, and G7167B multisampler linked to an AB Sciex API4000/API5500 mass spectrometer with electrospray ionization. All compounds were detected in positive or negative multiple reaction monitoring mode against internal standard. multiple reaction monitoring transitions are listed in Supplemental Table 1. An Ascentis Express C18 column $(30 \times 2.1 \mathrm{~mm}$,
2.7- $\mu \mathrm{m}$ particle size) was used with mobile phases (A) water and (B) acetonitrile with either $0.1 \%$ acetic acid or $0.1 \%$ ammonia. Gradients for high-performance liquid chromatography are listed in Supplemental Table 2.

\section{Results}

Prediction of Lysosomal Drug Sequestration in Rat Hepatocytes. Our previous studies showed good predictability of lysosomal sequestration in rat hepatocytes for propranolol and imipramine (Schmitt et al., 2019). To further confirm the reliability of these predictions, the distribution into lysosomes of rat hepatocytes was measured experimentally and predicted in silico for a broad set of compounds. These PKIs comprised a broad range of physicochemical properties. With a log

TABLE 2

Concentration of BMP in rat tissues with corresponding fractional volumes of lysosomes

Liver was taken as reference, whose lysosomal content was determined experimentally. Othe tissues were calculated relative to the liver by bis(monoacylglycero)phosphate tissue concentrations.

\begin{tabular}{lcc}
\hline Tissue & BMP Concentration $^{a}$ & $\begin{array}{c}\text { Fractional Tissue Volume of } \\
\text { Lysosomes (\%) }\end{array}$ \\
\hline Blood cells & $\begin{array}{c}\text { pmol/mg tissue } \\
\text { LLOQ }^{b}\end{array}$ & n.a. \\
Plasma & $<$ LLOQ $^{b}$ & n.a. \\
Adipose & $0.3 \pm 0.1$ & 0.03 \\
Brain & $15 \pm 1.9$ & 1.4 \\
Gut $^{c}$ & $14 \pm 6.8$ & 1.2 \\
Heart & $3.5 \pm 0.6$ & 0.32 \\
Intestine & $15 \pm 6.0$ & 1.4 \\
Intestine large & $9.6 \pm 1.9$ & 0.88 \\
Kidney & $19 \pm 2.6$ & 1.7 \\
Liver & $14 \pm 3.5$ & 1.3 \\
Lung & $17 \pm 5.7$ & 1.5 \\
Muscle & $1.2 \pm 0.1$ & 0.11 \\
Skin & $1.8 \pm 0.6$ & 0.16 \\
Spleen & $58 \pm 4.8$ & 5.3 \\
Stomach & $12 \pm 1.9$ & 1.1 \\
\hline
\end{tabular}

${ }^{a}$ Taken from Wang and Schmitt et al. (2019); data represent means \pm S.D.

${ }^{b}$ Lower limit of quantification.

${ }^{c}$ Gut represents the weighted mean of small and large intestine accounting for the $2.3 \times$ higher volume of the small intestine. 
P of 1.6-6.6, all compounds possessed enough lipophilicity to cross the lipid bilayer by passive diffusion, thus being able to reach the inner acidic compartment of lysosomes - a prerequisite to undergo lysosomal trapping. The basicity of compounds ranged from a $\mathrm{p} K_{\mathrm{a}}$ of 0.2 up to 9.7, with some of the compounds possessing a second weakly basic moiety. All physicochemical properties used to predict the lysosomal sequestration of the compounds are summarized in Supplemental Table 3.

The predictions for compounds not showing any tendency for lysosomal trapping were correctly confirmed by the experimental results (Fig. 2). Strong lysosomal accumulation ( $\geq 50 \%$ ) was well predicted, with only slight deviations from the experimental results. Only saracatinib, with $50 \%$ of the drug trapped in lysosomes, was markedly below the predicted $69 \%$. As lysosomal trapping increases exponentially in the range of $\mathrm{p} K_{\mathrm{a}} \sim 6.5-7.5$, predictions of moderately lysosomotropic compounds ( $\sim 20 \%-40 \%$ in lysosomes) showed higher deviations from experimental results with increasing basicity. Besides good predictions for gefitinib and dasatinib, crizotinib and tandutinib were both overpredicted by about $25 \%$, and lapatinib was underpredicted by 24\% (Fig. 2A). Statistical analysis showed a linear relationship between predicted and experimentally determined lysosomal sequestration for this diverse set of compounds, with a correlation coefficient of $r^{2}=0.8$ and no systematic over- or underprediction (Fig. 2B). In total, $68 \%$ of the compounds had a total deviation of $<10 \%$ and $86 \%$ of $<15 \%$. With lysosomes having a significant influence on intracellular distribution of basic lipophilic drugs, the incorporation of $\mathrm{pH}$-driven sequestration into the mechanistic distribution equation of Rodgers et al. (2005) should further improve prediction results.

Estimation of the Size of the Endo-/Lysosomal System in Rat Tissues via BMP Content. Lysosomal content was calculated based on the lysosome-specific marker lipid BMP (see Materials and Methods section). The lysosomal content varies greatly between the different tissues. Adipose, muscle, and skin tissue have only a little BMP content, which calculates to few lysosomes with fractional tissue volumes between $0.03 \%$ and $0.16 \%$, followed by the heart with $0.32 \%$. Most tissues have a similar modest calculated fractional volume of lysosomes as the liver (about 1\%-1.7\%). The spleen, however, has by far the most lysosomes based on the relative BMP content, with a fractional volume of $5.3 \%$ (Table 2). For the first time, we have estimated the size of the lysosomal system across many body tissues. This fulfills the main prerequisite to be able to incorporate $\mathrm{pH}$-driven lysosomal sequestration in the mechanistic approach to predict drug tissue distribution.

Prediction of Drug Tissue Distribution Including Lysosomal Sequestration in all Rat Tissues. We have extended the mechanistic model by Rodgers and Rowland (2007) to predict drug tissue distribution by inclusion of tissue-specific lysosomal compartments. As the model assumes an equal distribution of the unprotonated free base

\section{TABLE 3}

Fold increase in predicted $\mathrm{Kpu}$ of 27 basic lipophilic drugs in rat tissues by incorporating lysosomal trapping into the mechanistic model compared with Rodgers et al. (2005) (eq. 2 vs. eq. 3)

\begin{tabular}{lc}
\hline Tissue & Fold Increase of Kpu Minimum to Maximum (mean) \\
\hline Adipose & $1.00-1.08(1.01)$ \\
Brain & $1.01-1.81(1.31)$ \\
Gut & $1.00-1.72(1.11)$ \\
Heart & $1.00-1.20(1.03)$ \\
Kidney & $1.00-2.09(1.11)$ \\
Liver & $1.00-1.74(1.08)$ \\
Lung & $1.00-1.94(1.11)$ \\
Muscle & $1.00-1.06(1.01)$ \\
Skin & $1.00-1.11(1.02)$ \\
Spleen & $1.01-4.09(1.43)$ \\
\hline
\end{tabular}

across all compartments, the pH-driven lysosomal partitioning can be calculated from plasma concentrations. For simplification, an effective lysosomal $\mathrm{pH}$ was derived from eq. 1 , which could reliably predict the lysosomal distribution of a large set of compounds in rat hepatocytes. The effective $\mathrm{pH}$ comprises the information gained from the full $\mathrm{pH}$ profile and should not be mistaken for the widely used average lysosomal $\mathrm{pH}$.

To examine the influence of this addition, the original data set of basic lipophilic drugs was predicted with the equation according to Rodgers et al. (2005) and the newly developed lysosomal extended eq. 3.

In all tissues the Kpu increased by considering lysosomal trapping in the predictions. However, in tissues with few lysosomes like fat, heart, muscle and skin the Kpu increased by only $1 \%-3 \%$ on average. Because of the considerably greater size of the endo-/lysosomal system in intestine, kidney, liver, and lung, predictions of Kpu increased by about $10 \%$ on average. With $29 \%$ and $43 \%$ for brain and spleen, respectively, lysosomal sequestration showed the biggest impact on those tissues (Table 3), showing a large spread depending on compound-specific properties. Nicotine, for instance, was predicted about 300\% higher in spleen and $100 \%$ higher in lung and kidney, whereas verapamil was not affected in any of these tissues. Although nicotine showed the greatest change in most of the tissues, procainamide and quinidine showed the strongest increase (80\%) in brain (Fig. 3). Interestingly, stronger differences in $\mathrm{Kpu}$ changes between compounds were seen in brain and spleen than in other lysosome-rich tissues, like kidney, liver, or lung. In tissues with few lysosomes, the Kpu prediction did not change notably for any compound. Deviations to experimental Kpu values did not significantly change for tissues with few lysosomes but improved for all lysosome-rich tissues compared with Rodgers et al. (2005). Detailed prediction results including Kpu values for all compounds across tissues are summarized in Supplemental Table 4. Incorporating lysosomal trapping into the mechanistic equation led to an overall improvement of the predictions for drug tissue partition.

Tissue Distribution of Protein Kinase Inhibitors. The effect of lysosomal trapping on tissue distribution was further examined with 13 PKIs, which showed moderate to strong lysosomal sequestration in rat hepatocytes and could successfully be predicted by eq. 1. Those compounds were predicted using the original equation by Rodgers et al. (2005) and the lysosomal extended eq. 3. Because of the lack of experimental tissue Kpu values for those compounds, the Vuss was used

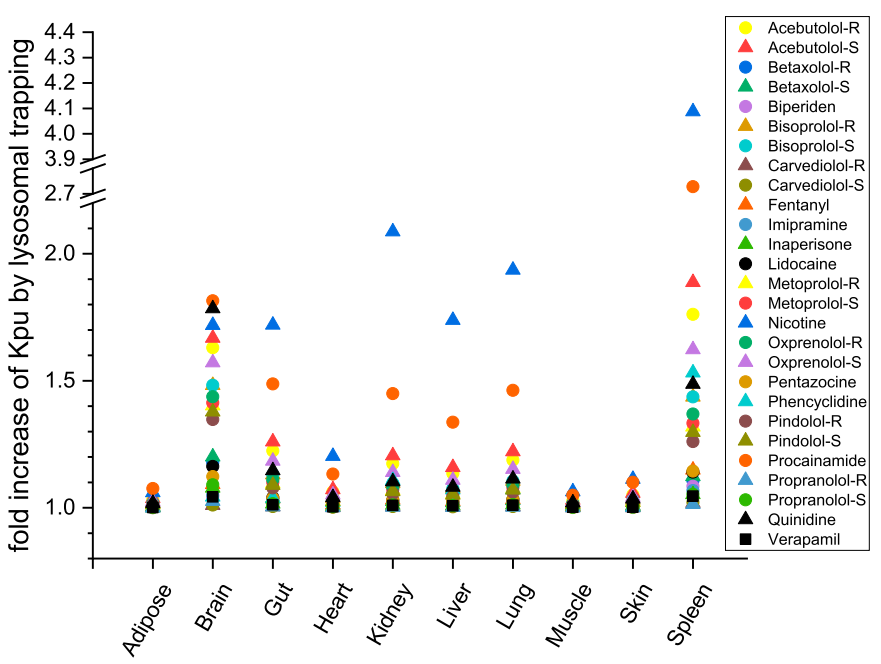

Fig. 3. Impact of the newly developed lysosomal trapping term on the prediction of tissue distribution of 27 basic lipophilic drugs. Data represent the fold increase of Kpu calculated by eq. 3 compared with predictions of Rodgers et al. (2005). Detailed results are summarized in Supplemental Table 4. 
TABLE 4

Compound-specific parameters, in vivo PK, and predicted Vuss of 13 protein kinase inhibitors

Physicochemical parameters were predicted using ADMET Predictor (Simulations Plus Inc.). Unbound fraction in plasma and blood-to-plasma concentration ratios were measured experimentally. In vivo Vss was obtained from literature or in vivo PK studies. The amount of intracellular drug located in lysosomes (\% in lysosomes) was calculated according to eq. 1 .

\begin{tabular}{|c|c|c|c|c|c|c|c|c|}
\hline Compound & $\mathrm{p} K_{\mathrm{a}}$ & $\begin{array}{c}\log \\
P\end{array}$ & $f u^{a}$ & $\mathrm{~B}: \mathrm{P}^{a}$ & $\begin{array}{l}\text { Vss } \\
\text { exp. }{ }^{b}\end{array}$ & $\begin{array}{l}\text { Vuss } \\
\text { exp. }^{c}\end{array}$ & $\begin{array}{l}\text { Vuss } \\
\text { pred. }^{d}\end{array}$ & $\begin{array}{c}\% \text { in } \\
\text { Lyososomes }\end{array}$ \\
\hline & & & & & $\mathrm{l} / \mathrm{kg}$ & $\mathrm{l} / \mathrm{kg}$ & $\mathrm{l} / \mathrm{kg}$ & \\
\hline Afatinib & $8.4,3.9$ & 3.8 & 0.11 & 2.6 & 16 & 152 & 224 & 54 \\
\hline Bosutinib & $8.4,4.1$ & 4.1 & 0.09 & 1.1 & 15 & 177 & 93 & 51 \\
\hline Cediranib & $9.1,3.4$ & 4.1 & 0.14 & 1.6 & 5.5 & 41 & 90 & 54 \\
\hline Crizotinib & $9.7,4.1$ & 3.6 & 0.04 & 0.94 & 2.9 & 82 & 130 & 57 \\
\hline Dasatinib & $6.9,3.9$ & 4.0 & 0.05 & 1.1 & 6.3 & 140 & 245 & 31 \\
\hline Gefitinib & $6.9,4.1$ & 3.8 & 0.04 & 0.8 & 9.2 & 259 & 142 & 31 \\
\hline Imatinib & $8.2,4.5$ & 4.4 & 0.02 & 0.86 & 2.6 & 165 & 288 & 60 \\
\hline Lapatinib & $6.5,4.1$ & 4.6 & 0.003 & 0.48 & 1.8 & 551 & 1085 & 20 \\
\hline Masitinib & $8.1,4.2$ & 5.0 & 0.03 & 0.83 & 6.2 & 236 & 411 & 55 \\
\hline Saracatinib & $8.1,5.0$ & 3.2 & 0.10 & 1.7 & 10 & 97 & 130 & 69 \\
\hline Sunitinib & 9.0 & 2.9 & 0.03 & 1.6 & 5.5 & 171 & 375 & 53 \\
\hline Tandutinib & $8.9,4.6$ & 4.3 & 0.37 & 2.8 & 47 & 127 & 76 & 62 \\
\hline Vandetanib & $8.8,4.2$ & 4.5 & 0.14 & 2.3 & 27 & 189 & 157 & 57 \\
\hline
\end{tabular}

${ }^{a}$ Sources: bosutinib, FDA (2012); dasatinib, Kamath et al. (2008); gefitinib, EMA (2008a); imatinib, O'Brien and Fallah Moghaddam (2013); lapatinib, O'Brien and Fallah Moghaddam (2013); sunitinib, EMA (2006).

${ }^{b}$ Sources: afatinib, EMA (2013); bosutinib, FDA (2012); crizotinib, PMDA (2012); dasatinib, Lombardo et al. (2004); gefitinib, McKillop et al. (2004); lapatinib, EMA (2008b); saracatinib, Hennequin et al. (2006); sunitinib, EMA (2006); vandetanib, TGA (2013).

${ }^{c}$ Calculated from experimental volume of distribution and fraction unbound in plasma.

${ }^{d}$ Detailed prediction results (i.e., Kpu of tissues) are summarized in Supplemental Table 5.

as a distribution parameter to assess the quality of predictions. The data were primarily collected from literature and complemented by in vivo PK studies (Table 4). The lipophilicity of the tested compounds ranged from $\log$ P 3-5, all exceeding the recommended lipophilicity of Rodgers and Rowland (2007) of $\log \mathrm{P}<3$. Additionally, the compounds showed a larger spread in their $\mathrm{B}: \mathrm{P}$ ratios compared with the original data set. With a B:P ratio of 0.48 , lapatinib hardly partitions into blood cells, whereas tandutinib (with a B:P ratio of 2.8) has a preference to distribute into blood cells.

The predictions for Vuss covered a wide range, from 76 up to 1085 $1 / \mathrm{kg}$. Tandutinib, cediranib, and bosutinib showed the lowest Vuss, with 76,90 , and $931 / \mathrm{kg}$, respectively. Most of the compounds were predicted to have Vuss between 100 and 300 1/kg. However, sunitinib, masitinib, and lapatinib exceeded this range, with 375,411 , and $1085 \mathrm{l} / \mathrm{kg}$, respectively (Table 4). The prediction of Vuss did not change notably by including lysosomal trapping into the predictions. Results predicted according to the lysosomal extended eq. 3 only differed by up to $11 / \mathrm{kg}$ compared with the model by Rodgers et al. (2005) (Supplemental Table 5). On average, predictions of Vuss deviated from experimental results only by 1.7 -fold, with cediranib and sunitinib showing the highest deviation of 2.2-fold (Fig. 6).

\section{Discussion}

Current distribution models do not, or only partially, consider lysosomal sequestration, which is particularly important for basic lipophilic drugs (Poulin and Theil, 2002; Rodgers et al., 2005, Assmus et al., 2017). To close this gap, we have validated our previously published in silico prediction of lysosomal trapping in rat hepatocytes (Schmitt et al., 2019) and used this approach, in combination with published BMP tissue levels, as marker lipid for the endo-/lysosomal system in the body tissues of the rat (Wang and Schmitt et al., 2019) to predict Kpu and Vuss for the original data set from Rodgers et al. (2005). Comparison of the results allowed the evaluation of the overall impact of lysosomal sequestration on these PK parameters. The predictivity of the approach was further examined using a set of more lipophilic PKIs whose physicochemical properties are beyond the previous range, reflecting a more recent drug-like space.
Prediction of Lysosomal Trapping. The more diverse data set in the present study (Fig. 2) confirms the high predictivity of our previously published approach to predict lysosomal trapping (Schmitt et al., 2019). As reported for propranolol and imipramine (Schmitt et al., 2019), the superiority of using the full $\mathrm{pH}$ profile of the endolysosomal system instead of simply using an average lysosomal $\mathrm{pH}$ value was also found for PKIs. The absolute deviation of predictions from experimental results was reduced from $12 \%$ to $6 \%$ (data not shown), and the number of underpredicted compounds went down by one-third from 18 to 12 . The underestimation for lapatinib (20\% instead of $44 \%$ ) may be explained by the predicted $\mathrm{p} K_{\mathrm{a}}=6.5$ that was used for lapatinib. An alternative software (Chemaxxon; Chemaxxon Ltd.), which estimates a $\mathrm{p} K_{\mathrm{a}}$ of 7.2 , would predict a lysosomal sequestration of $40 \%$, which is fully in line with the experimental observation and highlights the impact of the $\mathrm{p} K_{\mathrm{a}}$ estimates.

Lysosomal Sequestration in the Prediction of Drug Tissue Partition. With no quantified BMP in red blood cells, lysosomes are not expected in this cell type, which is in line with the absence of any information on lysosomes in an extensive review on red blood cells given by (Hinderling, 1997). Accordingly, the estimation of Ka, the association constant of the compound for acidic phospholipid from the blood-to-plasma ratio, as recommended by Rodgers et al. (2005), still holds. The incorporation of the lysosomal sequestration into the mechanistic equation revealed tissue-dependent effects on Kpu. Adipose tissue, muscle, and heart were minimally affected because of the low abundance of lysosomes. However, for lysosome-rich tissues, such as kidney, spleen, and liver, which are generally underpredicted by

TABLE 5

Composition of liver Kpu by subcellular distribution processes calculated by eq. 3 for nicotine and propranolol-R

\begin{tabular}{lcccccc}
\hline \multirow{2}{*}{ Compound } & \multicolumn{5}{c}{ Partial Liver Kpu } & $\begin{array}{c}\text { Liver } \\
\text { Kpu }\end{array}$ \\
\cline { 2 - 6 } & IW & EW & AP & NL/NP & Lyso & \\
\hline Nicotine & 1.2 & 0.2 & 0.0 & 0.1 & 1.1 & 2.5 \\
Propranolol-R & 1.4 & 0.2 & 668 & 0.7 & 1.5 & 671 \\
\hline
\end{tabular}

Lyso, lysosomes. 


\section{Impact of lysosomal trapping on Kpu and Vuss depends on the following properties:}

\section{High contribution:}

- Basicity $\uparrow$

- Lipophilicity $\downarrow$

- B:P-ratio $\downarrow$
Low contribution:

- Basicity $\downarrow$

- Lipophilicity $\uparrow$

- B:P-ratio $\uparrow$
Fig. 4. Compound properties for which a higher influence of lysosomal trapping on tissue distribution can be expected. Note: Applies to lipophilic bases, which passively diffuse across membranes in their unionized form.

Rodgers et al. (2005), up to 4-fold higher Kpu values (e.g., nicotine/ spleen) were observed, leading to improved predictions. In total about $27 \%$ (vs. $23 \%$ ) of the 245 predicted Kpu values were within 1.25 -fold of the experimental results. Predictions within 2-fold were achieved for $63 \%$ (vs. 60\%) of the Kpu values, whereas false predictions with deviations of greater than 4 -fold were reduced to $9 \%$ (vs. $11 \%$ ).

For compounds like nicotine, Kpu was strongly affected by lysosomal trapping, with a contribution of up to $50 \%$ of the liver Kpu (Table 5), suggesting significant partitioning into lysosomes and intracellular water but low tissue binding. These compounds share a high basicity, relatively low lipophilicity, and a low B:P ratio (Fig. 4). In contrast, tissue distribution of compounds with similar $\mathrm{p} K_{\mathrm{a}}$ but higher lipophilicity and B:P ratio is clearly dominated by lipid partitioning, in particular phospholipid binding with little overall contribution by lysosomal trapping. For example, despite showing strong lysosomal trapping, the Kpu of propranolol in the liver is almost entirely governed by its affinity for acidic phospholipids ( $>99 \%$, Table 5).

Despite the strong effect of lysosomal trapping on Kpu predictions for some compounds in some tissues, there was only a marginal impact on Vuss. On average, Vuss predictions increased by $3 \%$, thus not significantly changing compared with Rodgers et al. (2005) (Figs. 5 ). Lysosome-rich tissues in which $\mathrm{Kpu}$ changes were observed tend to have rather small tissue volumes (Table 1). Spleen, with only $0.83 \mathrm{ml}$ in rat, the tissue richest in lysosomes, does not contribute much to Vuss, as it represents only $\sim 0.3 \%$ of the body volume. Furthermore, tissue

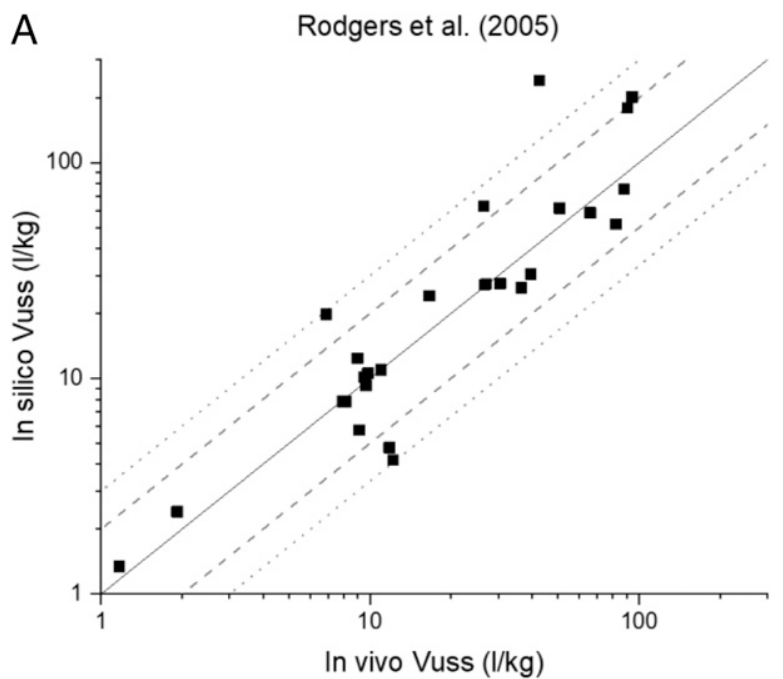

distribution depends not only on lysosomal trapping but also on tissue binding.

Our liver model does not take into account sequestration by Kupffer cells that may contain even more lysosomes than hepatocytes (Blouin et al., 1977). Despite their low abundance ( $\sim 2 \%$ of liver), they may also contribute to lysosomal sequestration by this organ. However, because data on their overall lysosomal content are not available, we could not include this cell type into our model. Furthermore, our model focuses on lysosomal trapping by $\mathrm{pH}$ partitioning and does not cover interactions of drugs with the lysosomal membrane, which may also be relevant (Colombo and Bertini, 1988; Hallifax and Houston, 2007). There are two key differences between the approach taken here and that taken by Assmus et al. (2017). First, although they cover lysosomal sequestration in lung, liver, and kidney, our method incorporates the lysosomal content of all tissues in the body, in particular spleen, brain, and gut, which are also rich in lysosomes. Second, the lysosomal sequestration as estimated by Assmus et al. is effectively driven by interactions with lysosomal membranes, thus emphasizing lysosomal binding, which is calculated analogously to the plasma membrane binding and hence tends to result in overpredictions as already reported by (Nigade et al., 2019). From our perspective, using the plasma membrane as a surrogate as applied by Assmus et al. is arguable, as the lysosomal membrane differs greatly in its composition (Amanuma-Muto et al., 1983; Kobayashi et al., 2002; Schulze et al., 2009). Furthermore, the acidic phospholipid phosphatidylserine, which is an important binding site for basic drugs, is located on the cytosolic side of the lysosomal membrane (HullinMatsuda et al., 2014) and is thus not able to interact with compounds inside of the lysosome. As no data on lysosomal membrane composition are available, our approach applies $\mathrm{pH}$-driven lysosomal trapping only. Lysosomal membrane binding can only be included once quantitative data of lysosomal membrane composition become available.

Tissue Distribution of Protein Kinase Inhibitors. We have selected this class of drugs to evaluate the performance of our model because their physicochemical properties, especially lipophilicity $(\log \mathrm{P}$ $=3-5$ ), are beyond the range of the original data set tested by Rodgers et al. (2005). Reliable predictions for such compound classes will help to guide lead optimization and facilitate PK projections to human (Reichel and Lienau, 2016; Petersson et al., 2019). The extended model was applied to predict the tissue Kpu and Vuss values of these mainly basic PKIs (Table 4). With intracellular distribution to lysosomes of up to $66 \%$ in hepatocytes, this mechanism is expected to have an influence

B

B Schmitt et al. (2020)

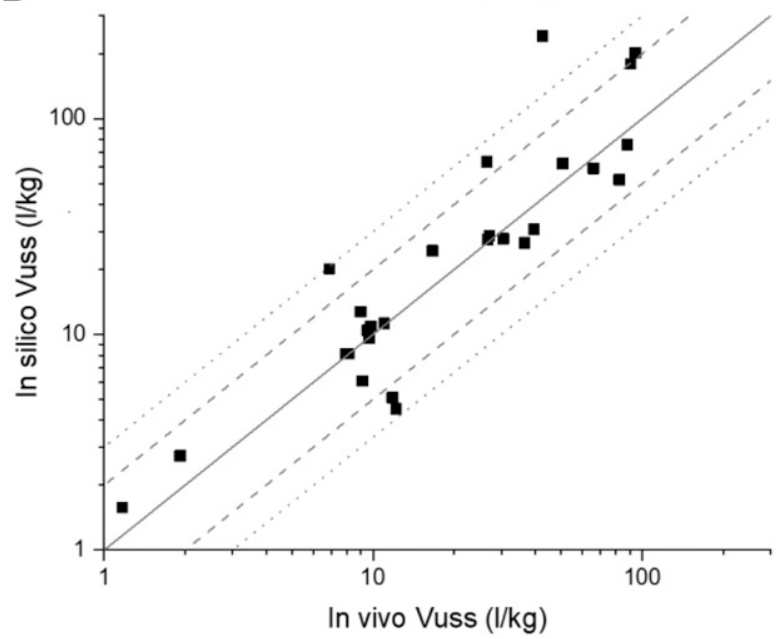

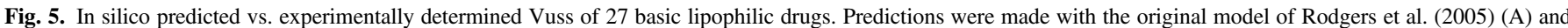
with the incorporation of lysosomal trapping (B). Lines represent a factor of 2 (dashes) and 3 (dots) on either side of the line of unity (solid). 


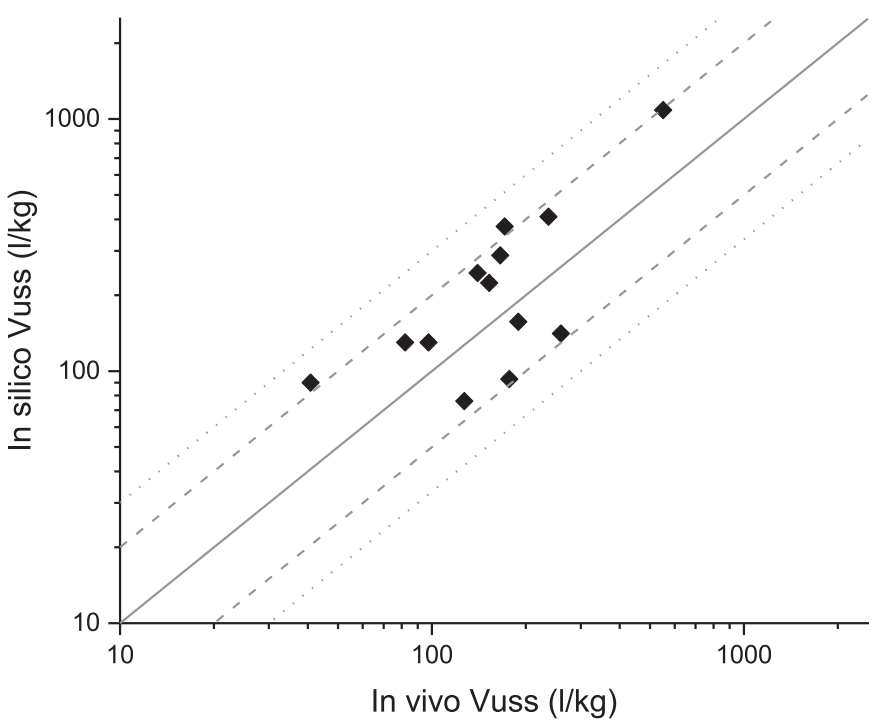

Fig. 6. Relationship between in silico predicted (eq. 3) and in vivo unbound volume of distribution in rats of 13 basic lipophilic protein kinase inhibitors. Lines represent a factor of 2 (dashes) and 3 (dots) on either side of the line of unity (solid).

on the intracellular drug distribution. At the level of the predicted $\mathrm{Kpu}$ values, however, there was no significant change across tissues compared with the model of Rodgers et al. (2005) (Supplemental Table 5). This highlights once more that although strong lysosomal trapping may occur, tissue partitioning of basic lipophilic compounds seems to be mainly driven by membrane binding, in particular to acidic phospholipids.

Although lysosomal trapping may not be significantly contributing to $\mathrm{Kpu}$ of all basic lipophilic drugs, it cannot be neglected as a mechanism of intracellular drug distribution ultimately affecting drug safety and efficacy (Smith et al., 2010, Guo et al., 2018). Indeed, the unbound intracellular concentrations that drive engagement of intracellular drug targets (Trünkle et al., 2020) can be influenced by lysosomal trapping, as recently demonstrated by Llanos et al. (2019), causing the prolonged activity of palbociclib.

\section{Conclusion}

We have extended the mechanistic, tissue composition-based equations to predict tissue distribution by inclusion of lysosomal compartments for all body tissues of the rat, thereby further improving the predictivity of Kpu for basic drugs undergoing lysosomal trapping. We see our model as another step in the continuing evolution of tissuedistribution models. It started with the seminal work by Poulin and Theil (2002) focusing on mechanisms related to the passive drug distribution of neutral molecules between aqueous/lipids/proteins in plasma and tissues and was subsequently advanced by Rodgers et al. (2005) to also include ionic interactions of charged molecules with acidic phospholipids and more recently by Assmus et al. (2017) to model lysosomal trapping in three organs. Our model extension now covers $\mathrm{pH}$-related lysosomal trapping in all major tissues of the rat and is likely extendable to other animal species, as well as to human. Direct comparison with the data set by Rodgers and Rowland (2007) showed an improvement of Kpu predictions, which, however, was smaller than expected for basic lipophilic drugs. This finding was confirmed for a set of PKIs showing that Kpu and Vuss were not significantly changed for these lipophilic basic compounds, mainly because of the very small lysosomal volume even in lysosome-rich tissues and the much stronger impact of lipid and acidic phospholipid binding to overall tissue distribution.

\section{Acknowledgments}

We would like to thank Stephan Menz for his assistance in mathematical questions and Daniel Trost and Martin Klein for their support in hepatocyte isolation and LC-MS/MS quantification. We are grateful to Malcolm Rowland for fruitful thoughts and discussions.

\section{Authorship Contributions}

Participated in research design: Schmitt, Reichel, Liu, Fricker, Lienau. Conducted experiments: Schmitt.

Performed data analysis: Schmitt, Reichel, Liu, Lienau.

Wrote or contributed to the writing of the manuscript: Schmitt, Reichel, Fricker, Lienau.

\section{References}

Amanuma-Muto K, Kanaseki T, Imanaka T, Ohkuma S, and Takano T (1983) Lipid composition of low-density lysosomal membrane fraction prepared from atheromatous aorta of cholesterolfed rabbits. Biochem Int 7:107-114.

Assmus F, Houston JB, and Galetin A (2017) Incorporation of lysosomal sequestration in the mechanistic model for prediction of tissue distribution of basic drugs. Eur J Pharm Sci 109: 419-430.

Bandyopadhyay D, Cyphersmith A, Zapata JA, Kim YJ, and Payne CK (2014) Lysosome transport as a function of lysosome diameter. PLoS One 9:e86847.

Banker MJ, Clark TH, and Williams JA (2003) Development and validation of a 96-well equilibrium dialysis apparatus for measuring plasma protein binding. J Pharm Sci 92:967-974.

Blouin A, Bolender RP, and Weibel ER (1977) Distribution of organelles and membranes between hepatocytes and nonhepatocytes in the rat liver parenchyma. A stereological study. J Cell Biol 72:441-455.

Chan R, De Bruyn T, Wright M, and Broccatelli F (2018) Comparing mechanistic and preclinical predictions of volume of distribution on a large set of drugs. Pharm Res 35:87.

Colombo MI and Bertini F (1988) Properties of binding sites for chloroquine in liver lysosomal membranes. J Cell Physiol 137:598-602.

de Duve C, de Barsy T, Poole B, Trouet A, Tulkens P, and Van Hoof F (1974) Commentary. Lysosomotropic agents. Biochem Pharmacol 23:2495-2531.

EMA (2006) Scientific discussion Sutent in Sunitinib, European Medicines Agency, Europa

EMA (2008a) Assessment report for Iressa, EMEA/CHMP/563746/2008, EMEA/H/C/001016, in Gefitinib, European Medicines Agency, Europa

EMA (2008b) Assessment report for Tyverb, EMEA/302222/2008, EMEA/H/C/795, in Lapatinib, European Medicines Agency, Europa.

EMA (2013) CHMP assessment report Giotrif, EMA/491185/2013, EMEA/H/C/002280, in Afatinib, European Medicines Agency, Europa.

FDA (2012) Pharmacology review(s) Bosulif, 2033410rig1s000, in Bosutinib, Food and Drug Administration, USA.

Gallala HD and Sandhoff K (2011) Biological function of the cellular lipid BMP-BMP as a key activator for cholesterol sorting and membrane digestion. Neurochem Res 36:1594-1600.

Guo Y, Chu X, Parrott NJ, Brouwer KLR, Hsu V, Nagar S, Matsson P, Sharma P, Snoeys J, Sugiyama Y, et al.; International Transporter Consortium (2018) Advancing predictions of tissue and intracellular drug concentrations using in vitro, imaging and physiologically based pharmacokinetic modeling approaches. Clin Pharmacol Ther 104:865-889.

Hallifax D and Houston JB (2007) Saturable uptake of lipophilic amine drugs into isolated hepatocytes: mechanisms and consequences for quantitative clearance prediction. Drug Metab Dispos 35:1325-1332.

Hennequin LF, Allen J, Breed J, Curwen J, Fennell M, Green TP, Lambert-van der Brempt C, Morgentin R, Norman RA, Olivier A, et al. (2006) N-(5-chloro-1,3-benzodioxol-4-yl)-7-[2-(4methylpiperazin-1-yl)ethoxy]-5- (tetrahydro-2H-pyran-4-yloxy)quinazolin-4-amine, a novel, highly selective, orally available, dual-specific c-Src/Abl kinase inhibitor. J Med Chem 49: 6465-6488.

Hinderling PH (1997) Red blood cells: a neglected compartment in pharmacokinetics and pharmacodynamics. Pharmacol Rev 49:279-295.

Hullin-Matsuda F, Taguchi T, Greimel P, and Kobayashi T (2014) Lipid compartmentalization in the endosome system. Semin Cell Dev Biol 31:48-56.

Kamath AV, Wang J, Lee FY, and Marathe PH (2008) Preclinical pharmacokinetics and in vitro metabolism of dasatinib (BMS-354825): a potent oral multi-targeted kinase inhibitor against SRC and BCR-ABL. Cancer Chemother Pharmacol 61:365-376.

Kobayashi T, Beuchat MH, Chevallier J, Makino A, Mayran N, Escola JM, Lebrand C, Cosson P, Kobayashi T, and Gruenberg J (2002) Separation and characterization of late endosomal membrane domains. J Biol Chem 277:32157-32164.

Llanos S, Megias D, Blanco-Aparicio C, Hernández-Encinas E, Rovira M, Pietrocola F, and Serrano M (2019) Lysosomal trapping of palbociclib and its functional implications. Oncogene 38:3886-3902.

Lombardo LJ, Lee FY, Chen P, Norris D, Barrish JC, Behnia K, Castaneda S, Cornelius LA, Das J, Doweyko AM, et al. (2004) Discovery of N-(2-chloro-6-methyl- phenyl)-2-(6-(4-(2-hydroxyethyl)- piperazin-1-yl)-2-methylpyrimidin-4- ylamino)thiazole-5-carboxamide (BMS-354825), a dual Src/Abl kinase inhibitor with potent antitumor activity in preclinical assays. J Med Chem 47:6658-6661.

MacIntyre AC and Cutler DJ (1988a) The potential role of lysosomes in tissue distribution of weak bases. Biopharm Drug Dispos 9:513-526.

MacIntyre AC and Cutler DJ (1988b) Role of lysosomes in hepatic accumulation of chloroquine. J Pharm Sci 77:196-199.

McKillop D, Partridge EA, Hutchison M, Rhead SA, Parry AC, Bardsley J, Woodman HM, and Swaisland HC (2004) Pharmacokinetics of gefitinib, an epidermal growth factor receptor tyrosine kinase inhibitor, in rat and dog. Xenobiotica 34:901-915.

Nigade PB, Gundu J, Pai KS, Nemmani KVS, and Talwar R (2019) Prediction of volume of distribution in preclinical species and humans: application of simplified physiologically based algorithms. Xenobiotica 49:528-539. 
O'Brien Z and Fallah Moghaddam M (2013) Small molecule kinase inhibitors approved by the FDA from 2000 to 2011: a systematic review of preclinical ADME data. Expert Opin Drug Metab Toxicol 9:1597-1612.

Petersson C, Papasouliotis O, Lecomte M, Badolo L, and Dolgos H (2019) Prediction of volume of distribution in humans: analysis of eight methods and their application in drug discovery. Xenobiotica 50:270-279.

PMDA (2012) Review report: xalkori capsules $200 \mathrm{mg}$ and $250 \mathrm{mg}$, in Crizotinib, Pharmaceuticals and Medical Devices Agency, Japan.

Poulin P and Theil FP (2000) A priori prediction of tissue:plasma partition coefficients of drugs to facilitate the use of physiologically-based pharmacokinetic models in drug discovery. J Pharm Sci 89:16-35.

Poulin P and Theil FP (2002) Prediction of pharmacokinetics prior to in vivo studies. II. Generic physiologically based pharmacokinetic models of drug disposition. J Pharm Sci 91:1358-1370. Reichel A and Lienau P (2016) Pharmacokinetics in drug discovery: an exposure-centred approach to optimising and predicting drug efficacy and safety. Handb Exp Pharmacol 232:235-260.

Rodgers T, Leahy D, and Rowland M (2005) Physiologically based pharmacokinetic modeling 1 : predicting the tissue distribution of moderate-to-strong bases. J Pharm Sci 94:1259-1276.

Rodgers T and Rowland M (2006) Physiologically based pharmacokinetic modelling 2: predicting the tissue distribution of acids, very weak bases, neutrals and zwitterions. J Pharm Sci 95 $1238-1257$.

Rodgers T and Rowland M (2007) Mechanistic approaches to volume of distribution predictions: understanding the processes. Pharm Res 24:918-933.

Schmitt MV, Lienau P, Fricker G, and Reichel A (2019) Quantitation of lysosomal trapping of basic lipophilic compounds using in vitro assays and in silico predictions based on the determination of the full $\mathrm{pH}$ profile of the endo-/lysosomal system in rat hepatocytes. Drug Metab Dispos 47:49-57.

Schulze H, Kolter T, and Sandhoff K (2009) Principles of lysosomal membrane degradation: cellular topology and biochemistry of lysosomal lipid degradation. Biochim Biophys Acta 1793 674-683.

Smith DA, Di L, and Kerns EH (2010) The effect of plasma protein binding on in vivo efficacy: misconceptions in drug discovery. Nat Rev Drug Discov 9:929-939.

TGA (2013) Australian Public Assessment Report for vandetanib, PM-2011-03002-3-4, in Vandetanib, Therapeutic Goods Administration, Australien.

Trünkle C, Lechner C, Korr D, Bouché L, Barak N, Fernández-Montalván A, Süssmuth RD, and Reichel A (2020) Concentration dependence of the unbound partition coefficient $\mathrm{Kp}_{\mathrm{uu}}$ and its application to correct for exposure-related discrepancies between biochemical and cellular potency of KAT6A inhibitors. Drug Metab Dispos 48:553-562.

Wang X, Schmitt MV, Xu L, Jiao Y, Guo L, Lienau P, Reichel A, and Liu X (2019) Quantitative molecular tissue atlas of Bis(monoacylglycero)phosphate and phosphatidylglycerol membrane lipids in rodent organs generated by methylation assisted high resolution mass spectrometry. Anal Chim Acta 1084:60-70.

Yata N, Toyoda T, Murakami T, Nishiura A, and Higashi Y (1990) Phosphatidylserine as a determinant for the tissue distribution of weakly basic drugs in rats. Pharm Res 7:1019-1025.

Address correspondence to: Dr. Philip Lienau, Bayer AG, Bldg. S116, 550, 13342 Berlin, Germany. E-mail: philip.lienau@bayer.com 